\title{
Disruptions to Transportation Networks: A Review
}

\author{
Shanjiang Zhu* David Levinson ${ }^{\dagger}$
}

May 9, 2011

\begin{abstract}
Travel decisions may be very stable in a familiar environment. Major network disruptions such as the I-35W bridge collapse disrupt habitual behavior. Such "natural" experiments provide unique opportunities for behavioral studies, but the time window for such studies is limited. A well-developed methodology is crucial for both data collection and analysis, and thus the soundness of behavioral models, especially in such a limited time window. Therefore, this paper reviews both theoretical and empirical studies on traffic and behavioral impacts of network disruptions. Findings from this paper offers prospective ideas about capturing the impacts of network disruption.
\end{abstract}

Keywords: Network disruption; Travel survey; Travel behavior

\section{Introduction}

The collapse, on August 1, 2007, of the I-35W bridge over the Mississippi

River in Minneapolis, abruptly interrupted the usual route of about 140,000

${ }^{*}$ University of Minnesota, Department of Civil Engineering, 500 Pillsbury Drive SE, Minneapolis, MN 55455 USA zhuxx120@umn.edu http://nexus.umn.edu

†dlevinson@umn.edu 
daily vehicle trips and substantially disturbed the flow pattern of the network. In addition to the heavy losses in life and injury, the network disruption also significantly impacted road-users and reshaped travel patterns in the Twin Cities area, which generated significant cost due to longer travel distance, higher levels of congestion, and the resulting opportunity losses. According to Minnesota Department of Transportation (MnDOT), rerouting alone could cost individual travelers and commercial vehicles $\$ 400,000$ daily based on Metropolitan Council planning model. Xie and Levinson (2008) find a lower, but still large, estimate of expected costs to road users, between $\$ 71,000$ and $\$ 220,000$ per day. As a result, a significant financial incentive was given to the contractor for the early completion of the replacement bridge. A similar financial incentive was employed after the Northridge Earthquake in California (the transportation-related costs due to network disruption in Los Angeles basin exceeded $\$ 1.6$ million per day (Wesemann et al., 1996) ) and a contractor earned $\$ 14.8$ million $(\$ 200,000$ per day) for completing work on freeway I-10 66 days ahead of initial schedule. Most of these decisions were based on planning models and conclusions were drawn through travel demand assignments on degraded networks, using User Equilibrium (UE) assumptions (assuming "the journey times in all routes actually used are equal and less than those which would be experienced by a single vehicle on any unused route" according to Wardrop (1952)). However, behavioral responses to the network disruption are much richer than what could be predicted by planning models. The network disruption forced travelers to explore the network and 
adjust their travel behavior according to their travel experience and external information resources. Immediately after the network disruption, travelers may:

- change their normal route because of road and ramp closure or congestion caused by traffic reallocation,

- adjust travel time to avoid congestion,

- satisfy needs at other destinations,

- consolidate trips (e.g. improving travel plans with trip chaining) and travel less frequently and more efficiently,

- switch to alternative travel modes,

- share travel duties among family members.

In the long term, travelers may also adjust their residential and work locations (Cairnes S. and Goodwin, 2002; Goodwin, 1977) . Until a new equilibrium is found (a period sometimes referred to as "the transient phase"), traffic may significantly deviate from the results predicted by planning models. For example, Clegg (2007) showed that a capacity reduction due to road construction generated an initial "over-reaction" effect followed by a "settling down" effect, using license plate match data from the city of York, England. Oscillation of overall traffic and individual route choices was reported. Although network disruptions are mostly temporary as damage is eventually 
repaired and capacity restored, travel experience accumulated during this time period could lead to permanent changes in travel patterns. van Exel and Rietveld (2001) indicated new patterns could become habitual once travelers explore and accept the driving experience during transit strikes.Cairnes S. and Goodwin (2002) also argued travel behaviors were conditioned on new experience instead of past history after investigating 70 case studies of road capacity reduction. Most of these day-to-day dynamics in travel demand cannot be captured by aggregate UE models Cairnes S. and Goodwin (2002). A good understanding of the behavioral changes and decision-making mechanism could not only better assist traffic management and the design of a mitigation plan in response of network disruptions, but also inform future research in travel demand modeling.

However, it is not easy to capture such a day-to-day learning and decisionmaking process. In an environment with which they are familiar, travelers' route choice decisions may be very stable. Goodwin (1977) argued travelers do not carefully and deliberately evaluate their choices because of "a reluctance to upset an ordered and well-understood routine". As the travel pattern remains unchanged, the role of habit increases and rational factors become less dominant, preventing relevant information from reaching decision makers and rational choices. Major network disruptions such as the I-35W bridge collapse could disrupt habitual behavior . Evidence suggests it took several weeks for the network to re-equilibrate (Zhu et al., n.d.), during which period, travelers continued to learn and adjust their travel decisions. 
These natural experiments provide unique opportunities to investigate how travelers valued different alternatives and made travel decisions over time.

Network disruptions, both planned and unplanned, are unusual but not unknown. Unplanned disruptions could be caused by natural disasters (e.g. tsunamis, earthquakes, floods, landslides, hurricanes), terrorist attacks (e.g 9/11), infrastructure failures (e.g. I-35W bridge collapse), severe accidents, etc. Examples of planned disruptions include road or ramp closure due to maintenance or construction work, transit strikes (e.g. 2005 transit strike at the New York City), major events such as Olympic Games and political conventions. These disruptions vary significant in both spatial and temporal dimensions. A strike by local transit workers may end in several days and its impacts are limited to the area they served. A severe earthquake may damage many links simultaneously, which may take years to rebuild. Because of inertia in travel behavior and inherent fluctuations in travel patterns due to ever-evolving network conditions, only significant disruptions to the network exhibit detectable changes on travel behavior, and thus on the aggregate traffic pattern. "Natural" experiments such as I-35W bridge collapse provide unique opportunities for behavioral studies, but the time window for such studies is limited because 1) capacity may be quickly restored by transportation agencies; 2) the economic and social background may change significantly over a longer time, preventing us from establishing any convincing causal effects. A well-developed methodology is crucial for both data collection and analysis, and thus the soundness of behavioral 
models, especially in such a limited time window.

This paper reviews both theoretical and empirical studies on traffic and behavioral impacts of network disruptions. This paper begins by summarizing types of transportation system effeects observed and conclusions drawn regarding demand responses. It then summarizes literature about specific behavioral changes. Then this paper focuses on the methods of data collection and analysis employed. Comparisons are made regarding the advantage and disadvantage of different research approaches in capturing various facets of travel behavior. The final section summarizes the previous discussion and offers some prospective ideas about capturing the impacts of network disruption.

\section{System effects}

Although there is a vast literature on travel behavior, research on behavioral responses to major network disruptions is limited (Giuliano and Golob, 1998). Large-scale network disruptions are unusual but not unknown. For bridge failure alone, we have in recent years seen the collapse of the I-80 San Francisco-Oakland Bay Bridge and I-880 Cypress Street Viaduct in Loma Prieta Earthquake, the Hatchie River Bridge in Tennessee, and the I-40 bridge at Webbers Falls, Oklahoma, among others. The lack of behavioral studies may arise from the difficulty of large-scale data collection after major incidents, especially when traffic monitoring devices such as loop detectors 
and cameras were not widely deployed. For example, the collapse, in 1975, of Tasman bridge in Hobart, Australia, significantly disrupted the network because the nearest alternative, the Bridgewater bridge, required 50 kilometers extra drive and there was little vehicular ferry service available. During the 14 months of reconstruction, of the 44,000 daily trips before the bridge collapse, 60\% disappeared (Hunt et al., 2002), creating a major pattern shift. However, no detailed analysis on behavioral changes was provided in the literature.

Table 1 summarizes 16 existing studies on behavioral responses after network disruptions in the literature. Some of them focused on one specific aspect of behavioral changes (e.g. Ferguson (1992) focused on transit riders), while others were more comprehensive and addressed a wide spectrum of issues in travel demand (e.g. Giuliano and Golob (1998)). Network disruptions caused by different types of incidents exhibited very different effects in travel demand (e.g. route switching may be the most universal after a bridge closure (Hunt et al., 2002; Zhu et al., n.d.), while responses to earthquakes have been more diverse), while the underlying behavioral pattern may be quite similar. Therefore, this section will provide a brief review of existing studies on network disruptions by their causes:

- transit strikes (summarized in Table 2)

- bridge closures (summarized in Table 3)

- special events, and 
- earthquakes (summarized in Table 3).

\subsection{Transit strike}

Public transit strikes disrupt the normal travel of transit riders and disturb the network by increasing use of personal vehicles. Transit strikes also provide a unique opportunity to understand alternatives transit riders have and how travel decisions are made, both of which are crucial for drafting future transportation policies. Although news coverage and qualitative descriptions about transit strikes are widely seen in the media, quantitative analysis of traffic and behavioral responses are limited.

The 1966 transit strike in New York City (lasting 13 days) significantly affected the network because public transit represented $60 \%$ of total trips in New York City. According to a study by the New York City Transit Au-

thority (NYCTA) based on home interviews of 8000 transit users, $67 \%$ of commuters switched to private vehicles, $75 \%$ as drivers and $25 \%$ as passengers. On the first day $50 \%$ travelers cancelled their trips but this number reduced to $10 \%$ in following days, showing the effects of initial shock and subsequent adaptations among travelers. With more cars in motion, the peak period spread from 2 to 4 hours. More interestingly, estimates from subsequent studies indicated permanent losses in transit ridership (2.1\% for work trips, $2.6 \%$ for shopping trips, and $2.4 \%$ for other trip purposes) after service was restored. Similarly, the 1981 and 1986 Orange County transit strike in California reduced $15 \%$ to $20 \%$ of transit trips after the strike according 
to Ferguson (1992). However, the importance of these numbers should not be exaggerated because public transit only represented $2 \%$ of total trips in Orange County. Lo and Hall (2006) investigated the effects of the Los Angeles transit strike based on loop-detector data. They revealed that although overall traffic flow remained almost the same due to the small number of bus riders, the speed scheme clearly showed a spread of the morning peak hour and a higher level of congestion during the strike period. Individual behavior, however, was not discussed in this paper due to lack to data.

A more detailed study was provided by Blumstein and Miller (1983), focusing on the 1976 transit strike in Pittsburgh, where $60 \%$ of the commuters to the CBD used transit. Both traffic counts and survey data were employed in the analysis. A surge in total traffic (up about $40 \%$ on the first day and $20 \%$ after), vehicle occupancy (up 50\%), downtown garage usage (up about $10 \%$ ), and taxi revenue (up 9.9\%) were observed and there was a spread of the peak period. Two subsequent telephone surveys indicated that most previous transit users were dropped off by a non-commuter (presumably a spouse), while $10 \%$ and $28 \%$ of previous transit riders decided to drive alone and carpool, respectively.

The authors argued that the "dropped-off" trips explained most of the increases in total traffic and vehicle occupancy, and vehicle ownership played a key role in choosing alternative modes (households with no car or only one car were more likely to use "drop-off" compared to households with two or more cars). Impacts on travel patterns of previous single drivers were 
also reported, including switching route (18\%), departing earlier (65\%), and changing parking place (31\%). However, no modeling work was reported despite the abundance of data.

van Exel and Rietveld (2001) provided a comprehensive review of 13 major strikes in the public transit sector. Their impacts on traffic vary significantly, primarily depending on the importance of public transit among other modes. However, individual travel choices, constrained by long-term factors such as car ownership, working and residential location, seem more sensitive to the length and extent of such strikes.

\subsection{Bridge closure}

Bridge closure damages the network by completely shutting down one important link. Its impacts on traffic and travel behavior vary significantly, depending on alternatives available. The aforementioned case of Tasman bridge represents one extreme where alternatives are almost non-existent, causing severe disruption in normal travel. However, network redundancy is more common in metropolitan areas, where impacts of bridge closure should be less severe.

Hunt et al. (2002) evaluated travelers' responses to a 14 month long closure (from August, 1999) of the Center Street Bridge in the city of Calgary, Alberta, Canada, based on both traffic counts and results from a telephone survey. Traffic observations indicated a minor drop (4.4\%) in total daily trips and a 15-minute forward shift of the morning peak period. Public transit rid- 
ership increased by $6.6 \%$, while vehicle occupancy declined $1.5 \%$. The traffic count data, however, only included observations of two days, in May 1999 and May 2000, respectively. The limited data prevented them from drawing statistically significant conclusions. Moreover, background conditions may have changed significantly over a year, preventing them from establishing any convincing causal effects. Therefore, a telephone interview survey was conducted to supplement the study, which generally confirmed previous findings. Although route switching effects were reported (15\% to $30 \%$ of users of five parallel bridges before the bridge closure used a different bridge), no robust analysis was provided.

Clegg (2007) showed that a partial bridge closure (capacity significantly reduced) due to road construction generated an initial "over-reaction" effect followed by a "settling down" effect, using license plate match data from York, England. Oscillation of overall traffic and individual route choice were reported.

Zhu et al. (n.d.) and Zhu et al. (2011) (this volume) review traffic and behavioral effects of the collapse of the I-35W Mississippi River Bridge in Minneapolis, Minnesota. Both the survey data and traffic counts suggested that total travel demand did not significantly reduce after the network collapse, possibly because of redundant capacity provided by alternatives. However, the results suggest about 50,000 fewer vehicles were crossing the Mississippi River on a daily basis in the Twin Cities. The average total travel time is clearly longer on average for those commuting to downtown or the nearby 
University of Minnesota, two areas close to the I-35W bridge. The peak period on the I-94 bridge, a major freeway alternative, clearly spread. The bridge collapse generated a small increase in public transit ridership, which is consistent with observations in previous research (Giuliano and Golob, 1998).

\section{$2.3 \quad$ Special events}

Special events such as Olympic Games also significantly disrupt normal traffic by introducing a highly concentrated travel demand. However, transportation agencies usually have a greater authority in these circumstances and travelers are generally more willing to follow instructions. For exam-

ple, although promoting public transit is difficult, $74 \%$ trips were carried by public transit during 2004 Athens Olympics according to Dimitriou et al. (2006). High transit ridership was also observed during the 2000 Sydney Olympics according to(Hensher and Brewer, 2002) (no detailed percentage number provided), although bus riders had to wait as long as 45 minutes. As a result, background traffic dropped $2 \%$ to $4.5 \%$ depending on the location, and travel speed doubled. These events show great potential for public transit. Although questions on how to achieve similar transit usage in ordinary circumstances have been frequently asked, no detailed studies on decisionmaking mechanism under these circumstances have been provided. 


\subsection{Earthquakes}

Chang and Nojima (2001) investigated the post-disaster transportation system performance after the 1995 Kobe, 1989 Loma Prieta, and 1994 Northridge earthquakes, using measures based on length of network open, total and areal accessibility. No analysis on behavioral responses were provided. Instead, Tsuchida and Wilshusen (1991) investigated the ride-sharing program in Santa Cruz County, California, which was mandated immediately after the Lima Prieta Earthquake and was removed after capacity was restored. Traffic changes, however, were not included.

Giuliano and Golob (1998) and Wesemann et al. (1996) study traffic and behavioral responses after the 1994 Northridge Earthquake in Los Angeles basin, California. Caltrans systematically documented the freeway traffic volume and Los Angeles Department of Transportation (LADOT) counted arterial traffic on a randomly chosen weekday each month. Metrolink collected all passenger counts by station and different bus operators had monthly passenger ridership by route. Vehicle occupancy was roughly estimated by the level of High Occupancy Vehicle (HOV) lane usage. Total demand (in person-trips) and shares of different modes were evaluated by the trips crossing the I-5 corridor screen line drawn between south of I-5/SR-14 junction and Balboa Blvd. The traffic on I-5 (the bridge at Gavin Canyon and the interchange between I-5 and State Route 14 collapsed) dropped 59\% immediately

due to lack of alternative. However, after restoring $70 \%$ of pre-earthquake capacity by implementing a series of mitigation project, traffic volume in- 
creased to $88 \%$ of pre-earthquake levels. After full capacity was restored in May 1994, total traffic increased quickly and went beyond the 1993 level in June by $1 \%$. Arterials still sustained significantly higher traffic compared to the pre-earthquake levels (carrying $10.85 \%$ of all daily trips crossing the screen line on I-5 corridor compared to the $3.62 \%$ before earthquake). The rail ridership (Metrolink) surged (carrying $9.64 \%$ of all daily trips on the I-5 corridor $)$ immediate after the earthquake, and then gradually reduced $(0.83 \%$ of total trips, compared to $0.21 \%$ before the earthquake).

Bus ridership remained flat ( $0.29 \%$ of all trips on the same corridor) during this period. Transit trips only accounted for $1.1 \%$ of total trips once pre-earthquake capacity was restored. Meanwhile, a telephone survey was conducted to sample 1000 workers in February 1994. Significant changes were reported in all aspects of travel decisions, though with different magnitude. Changing route $(31.2 \%)$ and changing schedule $(21.7 \%$ of respondents left earlier while $7.9 \%$ left later) were the most dominant, while changing mode had a smaller but detectable proportion (5.8\% from drive alone to carpool/vanpool and $0.3 \%$ to transit). Similar trends were revealed on I-10 where the Fairfax Avenue bridge collapsed. Systematic data collection efforts from different transportation agencies allowed this study to evaluate changes in traffic patterns over time.

However, the traffic shares of freeway, arterials, and transit one month after full capacity were restored were still significantly different from the market shares one year before. And no arguments have been provided about 
whether traffic patterns had re-equilibrated, which is crucial for travel demand analysis. Duration of this re-equilibration process may extend from several days (Clegg, 2007) to one year (Hunt et al., 2002) depending on context, and in models this has usually been assumed without solid justification. Robust statistics have to be introduced to evaluate the equilibration process and longitudinal observations are required.

\section{Behavioral effects}

Behavioral responses after network disruptions are the key research question in all these studies, each of which had specific focuses depending on the context and data availability. Table 3 summarizes primary findings from the literature. Instead of chronologically reviewing these studies, this section presents important findings and unanswered questions where future research is needed.

\subsection{Route choice and departure time}

Cairnes S. and Goodwin (2002) investigated 70 case studies of road capacity reduction and concluded that although people changed mode, consolidated trips for different purposes and visited alternative destinations in response to network degradation, "changing route and changing journey time seem to be the most universal". Findings in the literature generally confirm this conclusion, while the magnitude of changes varies depending on the context. 
Although route switching effects were reported in these studies(Hunt et al., 2002), the details of actual routes used by respondents were ignored most of the time, preventing further theoretical studies. The survey methods used, including both telephone interview and mail-in questionnaires, cannot easily record and compare routes used, especially for car drivers. Ideally, automatic route recording devices such as GPS recorders should be employed in future research.

Identifying travel route using questionnaires is easier for transit users. Dimitriou et al. (2006) evaluated the travel pattern during 2004 Athens Olympics , using a survey of 14,000 Olympic Games passengers. The travel chains were analyzed, showing although visitors might drive a significant portion of entire trip, the mode for final stage was predominantly public transit. However, their study focused more on public transit planning during such one-time major events, while its implications for modeling individual travel decisions are limited.

\subsection{Mode shifts}

According to the stated preference survey conducted after reopening of I-880, $9 \%$ of respondents stated that they would considering moving further from work and $11 \%$ reported that they would consider taking a job further from home as a result of travel time savings. A small share (7\%) of respondents indicated that they would otherwise take transit if the bridge had not opened, which is surprisingly high. 
In the case of I-5 in California, $88 \%$ of traffic returned with only $70 \%$ of capacity restored. Therefore, travelers must search for extra capacity available in the previously off-peak period, and thus create new congestion. However, travelers still prefer to drive, even with an 11.7 to 21.7 minutes increase in delay. In the modern metropolitan area, network redundancy is very high. A tolerance as large as 20 minutes before switching mode implies that very few travelers would switch mode because of delay. Giuliano and Golob (1998) indicated that the parking shortages, crowdedness on trains, and delays due to frequently aftershock might drive many riders back to car. Also, accessibility provided by public transit is very low in decentralized Los Angeles.

\subsection{Travel experience}

Many researchers have argued travelers make travel decisions based on previous experience (Goodwin, 1977), which may introduce non-linearity and generate travel patterns in dis-equilibrium. van Exel and Rietveld (2001) indicated that strikes undermine the perceived reliability of public transit and encourage some transit riders to switch to driving alone or carpooling. Moreover, new patterns could become habitual once travelers consider the driving experience. Their conclusions are supported by evidence from the permanent losses in public transit ridership after major transit strikes, including 1966 New York City (2.1\%-2.6\%), 1977 Knoxville (7\%-16\%), 1981,1986 Orange County, CA (15\%-20\%), and 1995 Netherlands (0.3\%-2\%). 
Tsuchida and Wilshusen (1991) drew a similar conclusion after investigating the ride-sharing program in Santa Cruz County, California. Commuters were required to share vehicles during the reconstruction period after the Lima Prieta Earthquake. After the damage was repaired and ridesharing mandate removed, $57 \%$ of survey respondents continued with ride-sharing. More interestingly, the primary reason convincing them to continue was costsavings of ride-sharing experienced during this mandate (42\%), followed by the people they shared rides with (22\%), enjoyment of the trip (12\%), environmental preservation (12\%), and finally, less stress (10\%).

Hensher and Brewer (2002) noticed people were willing to change their behavior for a one-time "single largest major event" when evaluating performance of public transportation in 2000 Sydney Olympics, finding that background vehicle trips dropped and transit ridership was high. Priority

measures during the 2004 Athens Olympics increased the average speed of buses from $15-17 \mathrm{~km} / \mathrm{h}$ to $30-40 \mathrm{~km} / \mathrm{h}$, creating significant incentives for riding buses (Dimitriou et al., 2006). Both studies argued that travel experience and performance of public transportation during the Games could promote a permanent shift in travel pattern.

\section{Data collection}

High-quality data is crucial for empirical studies and it is a big challenge to design and implement data collection schemes within the limited time after 
network disruptions. Automatic data collection devices enable 24/7 traffic monitoring with higher accuracy, which could greatly expand the depth and extent of analysis. For example, longitudinal analyses were only implemented in the case of I-5 corridor after the Northridge Earthquake because Caltrans systematically documented freeway traffic data collected by loop-detectors, which was not available in many other studies. Data collection on arterials still depended on manual counts in all these studies, representing a major barrier for traffic analysis in the metropolitan area. This barrier could be overcome by retrieving traffic data from signal control systems, which has been widely deployed in major cities. HOV and HOT lanes provide good data resources for vehicle occupancy. However, we could not accurately estimate the vehicle occupancy on the entire network without supplementing typically collected data. Models have been proposed to estimate regional auto occupancy by using crash records (1996 in New York, 1998 in Connecticut, 2005 in Florida (Gan et al., 2005)), which are continuously collected and documented by transportation agencies. Although data for this approach are readily available in most states, they are usually biased because of over or under involvement of certain population groups in crashes. More research work is needed before these models could be widely applied to capture the usually small changes in auto occupancy after network disruptions. Similarly, ridership statistics from transit operators provide good estimates of total trips. However, it tells little about the boarding stops, boarding time and duration of those trips, all of which are crucial to fit a transit model. 
Traffic observations alone cannot support a well-founded analysis of behavioral changes. Well-administered surveys are need. In the literature, three types of surveys, telephone survey, home interview, and mail-in questionnaires, have been employed. Home-interview and telephone survey have higher response rate $((\geq 80 \%)$ in studies listed $)$, they are, however, also generally more expensive. Mail-in surveys have a much lower response rate in the literature. Moreover, concerns about self-selection biases should be addressed before using such data.

Plate matching was employed by Clegg (2007). By identifying vehicles at different survey points, trip travel time could be estimated. Based on the same approach, route choice could be systematically estimated. However, collecting license plate numbers is typically labor-intensive, and cannot be implemented on a large-scale without a major new infrastructure investment. Moreover, Clegg (2007) also reported that plate-matching is error-prone and more research is required to generate convincing results.

\section{Conclusion}

Although network disruptions occur from time to time and provide unique opportunities to explore travel behavior, existing studies in the literature are limited. Traffic data were limited in time and locations before loopdetectors were widely deployed, preventing continuous traffic observation. As a result, no statistical analyses have been allowed researchers to empirically 
measure the re-equilibration of traffic flow. A practical measure of network equilibrium could not only advance theoretical research in travel demand modeling, but also guide the efforts in survey and behavioral study.

Although surveys based on questionnaires, telephone calls, and home interviews have been routinely conducted and generated significant findings, they are not sufficient to assist recent research efforts in individual-based travel demand modeling. For example, none of the three preceding survey tools could provide a good description of route choices, which is crucial in large metropolitan areas because of the complexity in network and thus the large number of alternative routes. Moreover, although changes in departure time and route choices are frequently reported in surveys, they are seldom combined, preventing us from investigating these two choices as a whole. This combined model is attracting increasing interest in theoretical research.

Existing studies clearly show the important role of experience in travel decisions, which has been frequently discussed in theoretical studies. However, the barriers to empirically capture its role are two-fold. First, it is difficult to observe travel decisions over time with current survey approaches (respondents describe their travel pattern either on one day, or generally during a period). Second, it is very hard to integrate survey data with traffic information (predominantly from loop-detectors), which reveals the traffic environment travelers experienced.

Evidence from these studies provides strong arguments for introducing travel experience in demand modeling, which could not only improve accu- 
racy of demand forecasting, but also capture day-to-day traffic dynamics. More research is required to model travel experience and empirical studies after network disruptions could provide valuable guidance.

Considering these difficulties, more advanced survey approaches using Global Positioning System (GPS) to track travelers should be employed. Objective observations of travel decisions and experience such as route selected, departure time, travel speed, and on-route delay from these devices could supplement subjective evaluations collected from existing surveys, and thus allowing more sophisticated behavioral analysis. Moreover, devices such as GPS allow accurate observations of day-to-day route choices for the first time, and easily combine them with traffic information if clocks from both system are carefully synchronized. Such research initiatives could be very promising.

\section{Acknowledgments}

This material is based in part upon work supported by the National Science Foundation under Grant No. 0825768, BRIDGE: Behavioral Response to the I-35W Disruption: Gauging Equilibration and Grant No. 0753580, SGER: Responding to the Unexpected: Understanding Travelers' Behavioral Choices in the Wake of the Mississippi River Bridge Collapse; Minnesota Department of Transportation project Traffic Flow and Road User Impacts of the Col-

lapse of the I-35W Bridge over the Mississippi River; and the University of 
Minnesota Metropolitan Consortium. We would also like to thank Henry Liu, Kathleen Harder, John Bloomfield, Saif Jabari, and Adam Dancyzk. Any opinions, findings, and conclusions or recommendations expressed in this material are those of the authors and do not necessarily reflect the views of the National Science Foundation, Minnesota Department of Transportation.

\section{References}

Blumstein, A. and Miller, H. (1983), "Making do: The effects of a mass transit strike on travel behavior", Transportation, Vol. 11, Springer, pp. 361382.

Cairnes S., S. A. and Goodwin, P. (2002), "Disappearing traffic? the story so far", Proceedings of the Institution of Civil Engineers, pp. 13-22.

Chang, S. and Nojima, N. (2001), "Measuring post-disaster transportation system performance: the 1995 Kobe earthquake in comparative perspective", Transportation Research Part A, Vol. 35, Elsevier, pp. 475-494.

Clegg, R. (2007), "Empirical Studies on Road Traffic Responses to Capacity Reduction", Transportation and Traffic Theory 200\%: Papers Selected for Presentation at ISTTT17, Elsevier Science.

Dimitriou, D., Karlaftis, M., Kepaptsoglou, K. and Stathopoulos, M. (2006), 
"Public Transportation during the Athens 2004 Olympics: From Planning to Performance.", Transportation Research Board.

Ferguson, E. (1992), "Transit ridership, incident effects and public policy", Transportation research. Part A, Policy and practice, Vol. 26, Elsevier, pp. 393-407.

Gan, A., Jung, R., Liu, K., Li, X. and Sandoval, D. (2005), Vehicle Occupancy Data Collection Methods, Technical report, Florida International University. http://lctr.eng.fiu.edu/Documents/VehicleOccupancyFinalReport.pdf.

Giuliano, G. and Golob, J. (1998), "Impacts of the Northridge Earthquake on Transit and Highway Use", Journal of Transportation and Statistics, Vol. 1, pp. 1-20.

Goodwin, P. (1977), "Habit and Hysteresis in Mode Choice", Urban Studies , Vol. 14, Routledge, pp. 95-98.

Hensher, D. and Brewer, A. (2002), "Going for gold at the Sydney Olympics: how did transport perform?", Transport Reviews, Vol. 22, pp. 381-399.

Hunt, J., Brownlee, A. and Stefan, K. (2002), "Responses to Centre Street Bridge Closure: Where the" Disappearing" Travelers Went", Transportation Research Record, Vol. 1807, Trans Res Board, pp. 51-58.

Lo, S. and Hall, R. (2006), "Effects of the Los Angeles transit strike on 
highway congestion", Transportation Research Part A, Vol. 40, Elsevier, pp. 903-917.

Tsuchida, P. and Wilshusen, L. (1991), "Effects of the 1989 Loma Prieta Earthquake on Commute Behavoir in Santa Cruz County, California", Transportation Research Board.

van Exel, N. and Rietveld, P. (2001), "Public transport strikes and traveller behaviour", Transport Policy, Vol. 8, Elsevier, pp. 237-246.

Wardrop, J. (1952), Some theoretical aspects of road traffic research, in 'Proceedings of the Institute of Civil Engineers, Pt. II', Vol. 1, pp. 325-378.

Wesemann, L., Hamilton, T., Tabaie, S. and Bare, G. (1996), "Cost-of-Delay Studies for Freeway Closures Caused by Northridge Earthquake", Transportation Research Record, Vol. 1559, Trans Res Board, pp. 67-75.

Xie, F. and Levinson, D. (2008), "Evaluating the Effects of I-35W Bridge Collapse on Road-Users in the Twin Cities Metropolitan Region ", Transportation Planning and Technology, Vol. In Press.

Zhu, S., Levinson, D., Liu, H. and Harder, K. (n.d.), "The traffic and behavioral effects of the I-35W Mississippi River bridge collapse", Transportation Research Part A: Policy and Practice, Vol. 4(10).

Zhu, S., Tilahun, N., He, X. and Levinson, D. (2011), Travel Impacts and Adjustment Strategies of the Collapse and the Reopening of I-35W Bridge, 
in D. Levinson, H. Liu and M. Bell, eds, 'Network Reliability in Practice', Springer, pp. $\mathrm{xxx}-\mathrm{xxx}$. 


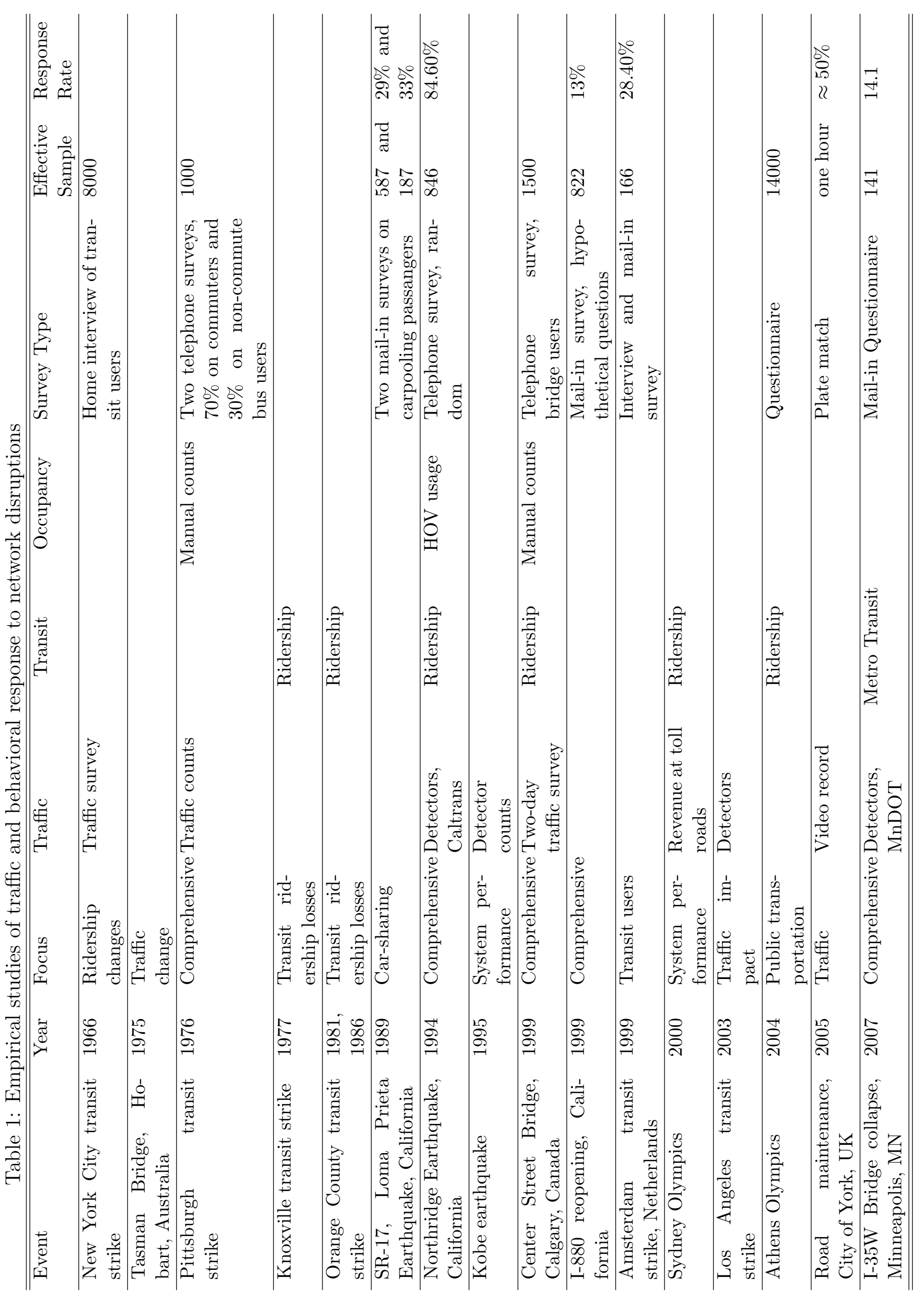




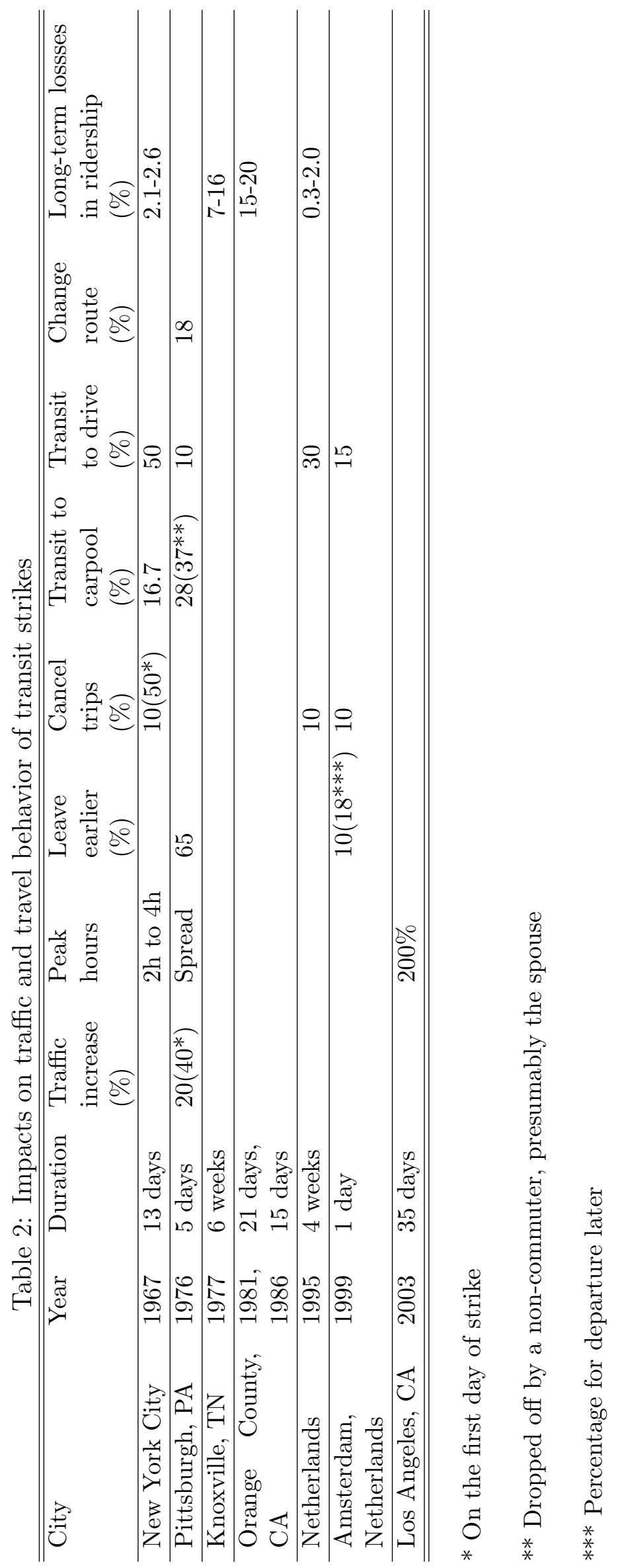




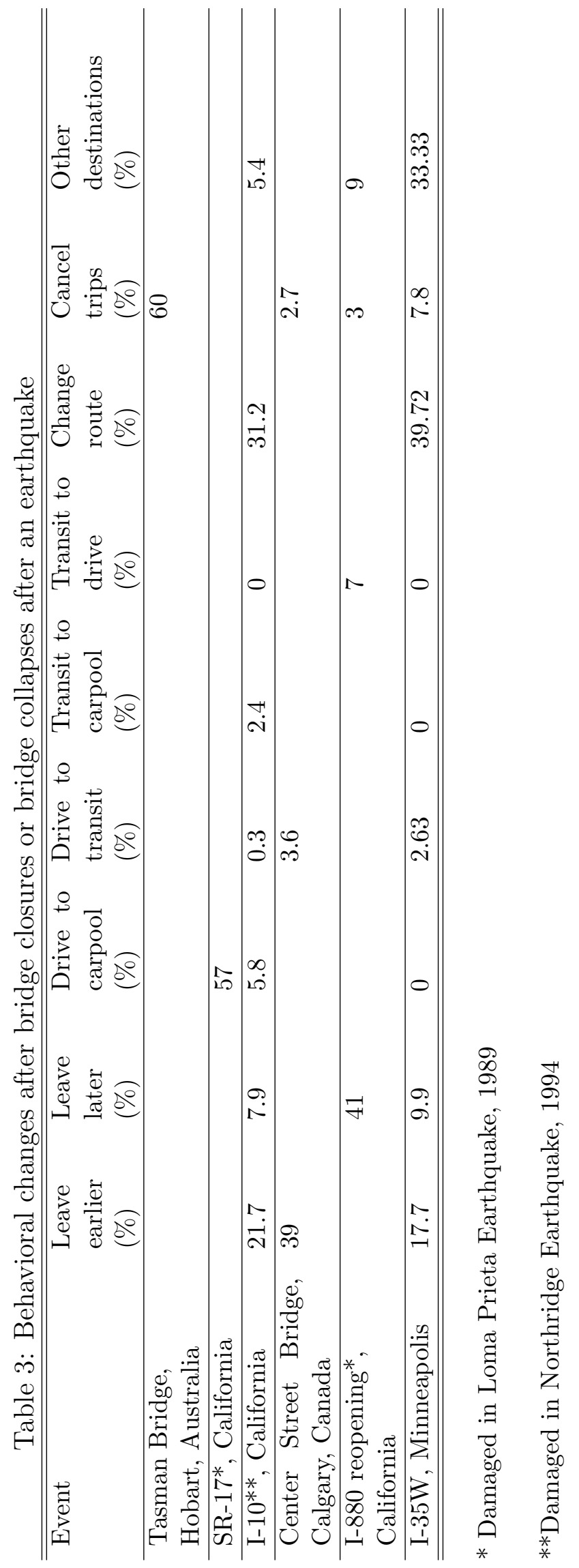

